\title{
Inaugural Target Discovery World Congress 2009
}

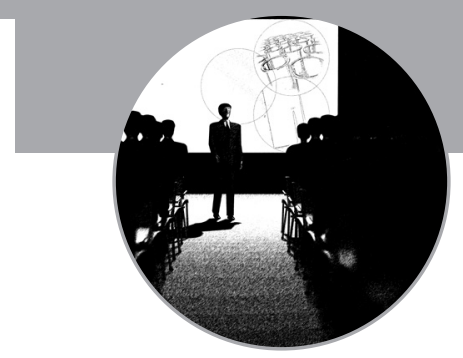

\author{
San Francisco, CA, USA, 4-5 August 2009
}

Target Discovery World Congress 2009 is the first of its kind in a series of trend-setting conferences conceived and organized by Select Biosciences. This was a multi-track event with a combined exhibition covering current potential drug targets, the platforms used to screen for hits and hit-to-lead methods. This conference included all the major drug-target classes: ion channels, protein kinases, G-protein-coupled receptors, nuclear receptors and ubiquitin pathways, as well as a special session devoted to screening platforms in academia. Each of the sessions started with a noteworthy keynote presentation by a world-class expert. There were a total of 44 high-quality podium presentations and 16 poster presentations.

The pharmaceutical industry is at a critical juncture now, with $\mathrm{R} \& \mathrm{D}$ costs escalating in leaps and bounds, and productivity, as indicated by investigational new drug filings, lagging far behind. This disconnect seems to be attributed to the difficulty in conquering the so-called 'valley of death', the Phase II clinical trials, where safety and efficacy of drug candidates are critically assessed. There is an almost $80 \%$ failure rate during Phase II clinical trials and almost half of this can be attributed to target-related failures. It is generally perceived that the pharmaceutical industry greatly underestimates the significance of patho/toxicobiology, and not well enough to pick the right therapeutic targets and, hence, the right drug candidates. It is academia, not the pharmaceutical industry, that is engaged in elucidating the mechanistic aspects of disease biology. In this context, the timing of the Target Discovery World Congress could not be overemphasized. The number of US FDA-approved drugs target less than $0.05 \%$ of genes, that is, 106 out of 25,500 in the human genome, and the rate of discovery for drugs that act on new therapeutic targets has been flat or on a downward trend for the last quarter of a century. Drug repurposing (finding new uses for old drugs) is the new mantra for many pharmaceutical companies. This new endeavor infers that, for many targets, there is more than one drug and that many drugs are promiscuous, targeting more than one protein. With more than US $\$ 50$ billion being spent on research each year, it is quite alarming to know that the pharmaceutical industry cannot confidently affirm the selectivity and specificity of the drugs it markets.
It is time to critically interrogate the druggable human genome to expand the target universe and facilitate the discovery of new, novel and safer drugs with greater selectivity and specificity. The conference keynote presentation by Rathnam Chaguturu (University of Kansas, KS, USA) stressed the need for both pharma and academia to engage in a partnership where the core missions of each institution are recognized, respected and accommodated, to conquer the so-called 'valley of death'. Instead of relying on small molecule-centric, forwardgenetic chemical approaches based on protein targets, he stressed the need for a paradigm shift where the focus is laid on genetic switches underlying the disease causing genome. PTC Therapeutics' ataluren targeting untranslated regulatory sequences of RNA is a prime example of such a groundbreaking endeavor.

\section{Track I: screening methods \& techniques}

The session brought together the Directors of several academic screening centers as well as faculty involved in ground-breaking research related to screening technologies. The session started with a keynote presentation by Rathnam Chaguturu. He discussed the drug-discovery and development initiatives at the University of Kansas fostering a matrix-team approach, a pharmaceutical industry-based best practice, in bridging the gap between in vitro drug target screening and in vivo validation, so as to make drug discovery in academia a reality.

Thomas Chung (Burnham Institute for Medical Research, CA, USA) provided an overview of Burnham's experiences in the network, from the pilot Molecular Libraries Screening

Rathnam Chaguturu
High Throughput Screening
Laboratories, Molecular
Biosciences, University of
Kansas, Structural Biology
Center, 2I2I Simons Drive,
Room I059, Lawrence,
KS 66047, USA
Tel.: +I 785 864 I7I7
Fax: +I 785864814 I
Email: rathnam@ku.edu


Centers Network to the production phase of the Molecular Libraries Probe Production Centers Network phase in overcoming technical and organizational challenges to forming a worthwhile national resource for the academic, not-for-profit and commercial research organizations. With almost 1300 bioassays and over 35 million datapoints deposited into the PubChem database, the MLSCN/MLPCN programs of the NIH have identified less than 40 probes with submicromolar potency, thus illustrating the enormous difficulty in identifying probes against novel therapeutic targets. One could also attribute this to inefficiency in coordinating therapeutic biology with medicinal chemistry, a rare combination of skills in the biomedical science community that is more biology-oriented.

With governmental agencies such as the NIH in the USA and the Medical Research Council in the UK funding cutting-edge research in target discovery, there is an evergrowing need for new signal detection technologies and platforms to facilitate the associated probe discovery and development efforts. Alan Waggoner (Carnegie Mellon University, PA, USA) discussed his team's efforts in developing a tool kit of fluorescent probes (biosensors) for quantifying the protein-regulation pathways of living cells at high resolution. The biosensordevelopment program blends genetics, protein structure, nucleic acid structure and fluorescent dye chemistry. His biosensor platform consists of single-chain antibodies that bind fluorogen dyes with high affinity and large fluorescence increases, and holds huge promise for exploring biomolecular interactions. Larry Sklar (Director of the University of New Mexico Center for Molecular Discovery for the NIH Roadmap Molecular Libraries Initiative) described the unique capabilities of flow cytometry towards homogeneous detection of molecular assembly, analysis of binding affinity and subsecond resolution of binding kinetics, often with femtomole sensitivity. This renewed appreciation offers exceptional opportunities for flow cytometry relating to receptor assembly and pharmacology, as well as a range of screening applications. Brian Cunningham (University of Illinois, IL, USA) presented novel label-free high-throughput screening of small molecule-protein interactions, cytotoxicity and G-protein-coupled receptor (GPCR) agonism/antagonism using photonic crystal optical biosensors incorporated into standard format microplates.
Target validation is becoming much more important and relevant in overcoming the 'valley of death' and high-content assay technology has found its niche in addressing this growing concern. High-content screening (HCS) technology makes use of spatially or temporally resolved imaging of cells using fluorescent tags to measure intracellular changes at the organelle level. Presentations by Hakim Djaballah (Memorial Sloan Kettering Cancer Center, NY, USA), Susan Heynen-Genel (Burnham Institute, CA, USA) and Joseph Trask (Duke University Medical Center, NC, USA) affirmed that the HCS platform is now a mainstream screening technology platform. BioFocus ${ }^{\mathrm{TM}}$ now has an HCS platform for identifying inhibitors of cell migration in cancer metastasis using adenoviral knockdown in a wound-healing assay. HCS data analysis is still a herculean task, as exemplified by Vebjørn Ljosa's (Broad Institute of Harvard and MIT, MA, USA) talk, which describing the capabilities of CellProfiler ${ }^{\mathrm{TM}}$, an open-source software for measuring cells and organisms in large-scale microscopy image sets.

\section{Track 2: targeting protein kinases, nuclear receptors \& ubiquitin proteosome system - Kinases}

Keynote presentation by Andrei Gartel (University of Illinois, IL, USA) affirmed the paradigm shift advocated by Rathnam Chaguturu by showing the repression of the transcriptional activity of FoxM1, an attractive therapeutic target in cancer, as well as FoxM1 expression by thiazole antibiotics, siomycin A and thiostrepton. This new mode of regulation of FoxM1 is another successful example of targeting the genetic switch rather than modulating the activity of the expressed protein in achieving disease prevention.

With Gleevec ${ }^{\circledR}$ sales exceeding US $\$ 3$ billion, the relatively young field of targeting protein kinases for drug discovery has gained unprecedented momentum. The pharmaceutical industry is now heavily focused on this class of targets for the treatment of a number of diseases, especially cancer and CNS disorders. The first wave of Aurora kinase inhibitors are entering clinical trials (Spiros Linardopoulos, Institute for Cancer Research, London, UK), and lipid kinases are a new class of drug targets being pursued (Jen Pan, Broad Institute, CA, USA). With the ubiquitous presence of kinases in cancer cells, targeting the noncatalytic/allosteric domains seems 
to be key in achieving kinase selectivity and specificity, thereby minimizing clinical uncertainty (Jen Pan, Broad Institute, MA, USA, and Ariel Fernandez, Rice University, TX, USA). Wendell Wierenga (Ambit Biosciences, CA, USA) discussed the utility of KinomeScan ${ }^{\mathrm{TM}}$ in generating uniquely selective kinase inhibitors for various indications in oncology and autoimmune diseases.

\section{- Nuclear receptors \& ubiquitin pathway}

Nuclear receptors are ligand-activated transcription factors that include the receptors for steroid and thyroid hormones, retinoic acid, oxysterols and bile acids. A total of 48 members of this superfamily of receptors exist in humans, and a significant number of these have no identified ligands. The vast majority of nuclear receptors that have identified ligands are targets for marketed drugs that treat diseases including cancer, inflammation and metabolic syndrome. Thus, the remaining orphan members of the superfamily may be a rich source of targets for the development of novel therapeutics. A keynote presentation by Thomas Burris (Scripps Research Institute, CA, USA) highlighted these aspects of nuclear receptor biology and his team's success in identifying both physiological and synthetic ligands for several orphan nuclear receptors. Fredika Robertson (MD Anderson Cancer Center, TX, USA) has made the case for targeting histone deacetylases to reprogram breast cancer stem cells. Histone deacetylase inhibitors target the self-renewal and pleuripotency of breast cancer stem cells, while simultaneously reprogramming expression of such genes as estrogen receptors, resulting in the return of responsiveness to anti-estrogen therapy.

With the therapeutic validation of the ubiquitin-proteasome system, as evidenced by the marketing approval of bortezomib (Velcade ${ }^{\circledR} /$ PS-341) for the treatment of multiple myeloma, Suresh Kumar (Progenera Corporation) discussed the discovery and development of novel USP7 inhibitors that selectively inhibit the growth of human tumor cells but not normal cells.

\section{Track 3: targeting G-protein-coupled receptors \& cell signaling systems}

Keynote presentation by Jeffrey Conn (Vanderbilt University, TE, USA) clearly articulated an exciting new approach to the discovery of highly selective, allosteric activators and antagonists of metabotropic glutamate and muscarinic acetylcholine receptors, which have robust activity in multiple animal models that predict efficacy in treatment of CNS disorders. Milt Teitler (Albany Medical College, NY, USA) presented evidence for functional homodimers to explain rapid and potent drug-induced inactivation and reactivation of serotonin receptors, using risperidone, 9-OH-risperidone and other drugs as case studies.

At the technology front, Bing Xie (CisBio Ltd, MA, USA) talked about new homogenous time-resolved flourescence (HTRF) solutions for cell-surface receptors, by monitoring cAMP (adenylate cyclase) and IP (phospholipase C). The combination of Lumi $4^{\circledR}-\mathrm{Tb}$ cryptate chemistry and SNAP-TagTM-labeling technology broadens HTRF applications, such as providing a nonradioactive alternative in ligand-binding assays and comprehensive investigation in receptor mechanistics (internalization and dimerization). Alexei Yeliseev (NIH, USA) discussed the use of NMR spectroscopy to elucidate the structure of human cannabinoid receptor CB2, one of the most sought after pharmaceutical targets. Hans Brauner-Osbourne (University of Copenhagen, Denmark) reported the cloning and analysis of expression of a novel human family C G-protein-coupled receptor, termed GPRC6A. Homology modeling of the GPRC6A amino terminal domain based on the crystal structure of the metabotropic glutamate receptor subtype 1 predicted interaction with $\alpha$-amino acids, and was employed to rationally select potential ligands.

A number of therapeutic targets have been developed to address the problem of uncontrolled platelet activation leading to vessel occlusion and subsequent stroke. These targets have focused on direct inhibition of G-proteincoupled receptor and integrin receptors as well as the inhibition of receptor ligands, resulting in an increase in bleeding, which, in some conditions, may be more deleterious to the patient than the clot itself. Michael Holinstat (Thomas Jefferson University, PA, USA) advocated a preferred therapeutic approach to treatment of platelet clot formation: to target signaling components downstream of receptor activation. Identification of these targets is a crucial first step in the development of novel therapeutics for the treatment of platelet clot formation with a minimal effect on bleeding. For example, as thrombin is the most potent activator in platelets, identification of targets downstream of its receptors, PAR1 and PAR4, which result in 
attenuation, but not complete, inhibition of platelet activation, would prove to be beneficial to maintaining normal hemostasis.

- lon channels as therapeutic targets

Ion channels are implicated in multiple diseases of excitable and nonexcitable tissues. The single biggest hurdle in exploiting ion channel targets for drug discovery has been the limitation of electrophysiology, the 'gold standard' tool for ion channel activity, with regards to its throughput capacity. Joe McGivern (Amgen, CA, USA) has overcome this insurmountable limitation by developing automated platforms for a number of voltage-gated ion channels, and used them in primary screening and in selectivity profiling. Lori Jin (Cerep, Paris, France) extended this concept in his poster presentation on the screening of $\mathrm{NaV} 1.5$ blockers using automated and conventional patch-clamp systems.

Temperature control during automated patch clamp assays is critical in studying temperature-sensitive ion channel targets. Cecilia Farre (Nanion, Munich, Germany) has developed a method for achieving rapid temperature changes around patch-clamped cells and has shown the method to be quite useful in characterizing heat inactivation of channels such as TRPV1. According to Narender Gavva (Amgen, CA, USA), members of the transient receptor potential (TRP) ion channel superfamily are proposed to mediate various sensory functions such as touch, pain, taste and thermosensation. TRP vanilloid (TRPV1-4), melastatin (TRPM8) family members and TRP ankyrin 1 (TRPA1) are directly activated by either heat or cold and by compounds that modulate sensations of warmth, cooling and burning pain. Agonists of TRPA1, TRPM8 and TRPV1 either cause pain and neurogenic inflammation or sensations of cold and pain in humans, suggesting that antagonists of these channels may be useful as analgesics. It appears that on-target undesirable effects are major hurdles for TRPV1 antagonists to become therapeutics. Preclinical data indicate that TRPA1 and TRPM8 may potentially be more attractive targets for analgesic discovery.
Heike Wulff (University of California, CA, USA) showed that calcium-activated potassium channel KCa3.1 plays an important role in the proliferation of dedifferentiated vascular discussed muscle cells, fibroblasts and lymphocytes. The KCa3.1 blockers were shown as potential new drugs to prevent restenosis, atherosclerosis and renal fibrosis. Jerod Denton (Vanderbilt University, TE, USA) discussed his group's recent discovery and characterization of small-molecule inhibitors of the renal outer medullary potassium channel and G-protein-coupled inwardly rectifying potassium channels. This fills the void with regards to the virtual absence of critical information on the molecular pharmacology of the inwardly rectifying $\mathrm{K}^{+}$channel family.

The poster presentations were all of high quality and relevance to the target discovery theme of the conference. The poster presentations dealt with new technology platforms (e.g., phage display, HCS and automated patch clamp systems), new therapeutic targets (GC binding factor 2 and transcription factors) and novel probes (triarylamines as connexin blockers). Stephanie Leavitt (Gilead, CA, USA) gave a well-received presentation on a fragment-based drug-discovery approach using the Corning $\mathrm{Epic}^{\circledR}$ system. In conjunction with the conference, two training courses, one dealing with fluorescence assays and the other on enzyme and binding assays in drug discovery, were presented by Jack Owicki.

The conference organizers are to be commended for addressing a timely topic, and bringing together the experts in the field to share their views on the current status of target discovery in finding new, novel and safer drugs.

\section{Financial \& competing interests disclosure}

The author has no relevant affiliations or financial involvement with any organization or entity with a financial interest in or financial conflict with the subject matter or materials discussed in the manuscript. This includes employment, consultancies, honoraria, stock ownership or options, expert testimony, grants or patents received or pending, or royalties.

No writing assistance was utilized in the production of this manuscript. 